\title{
AN EXTENSION OF THE THEOREM ON ALTERNATIVE DIVISION RINGS ${ }^{1}$
}

\author{
ERWIN KLEINFELD
}

1. Introduction. Familiarity with The structure of alternative division rings ${ }^{2}$ by $\mathrm{R}$. $\mathrm{H}$. Bruck and the author is assumed here. The symbol "BK" is used to refer to that paper. As this note is based on BK we shall avoid repetition by referring frequently to the appropriate theorems, lemmas, and identities of BK.

A ring is said to be simple if its only two-sided ideals are the zero ideal and the entire ring, that is, if it has no proper two-sided ideals. The main result of this note is the following:

Theorem M. Let $R$ be a simple, alternative ring without nilpotent elements and of characteristic not 2. Then $R$ is either associative or a Cayley-Dickson division algebra over its centre.

\section{Preliminaries.}

Lемма 2.1. Let $R$ be an alternative ring without nilpotent elements, generated by $x, y$, and $z$. Then $(x, y, z)^{2}$ lies in the centre of $R$.

Proof. The proof of this lemma is essentially contained in BK. All that need be shown is that $\left(u^{2}, x\right)=0$, where $u=(x, y, z)$. We begin with (BK 2.26) which states that $u\left(u^{2}, x\right)=0=\left(u^{2}, x\right) u$. Hence we get $\left(u^{2}, x\right)^{3}=\left(u^{2}, x\right) u(u, x)\left(u^{2}, x\right)+\left(u^{2}, x\right)(u, x) u\left(u^{2}, x\right)=0$, using Artin's Theorem. Thus $\left(u^{2}, x\right)=0$ and we are done.

The following theorem can be readily obtained from BK Lemma 4.1, using Lemma 2.1:

TheOREM 2.2. Let $R$ be an alternative ring with no nilpotent elements and of characteristic not 2 , generated by three elements $x, y$, and $z$ such that $(x, y, z) \neq 0$. Let $C$ be the centre of $R$. Then (i) $p x^{2}-q x+r=0$, for $p, q, r$ in $C, p \neq 0, r \neq 0$, (ii) $(x, y) \neq 0$.

The next lemma is concerned with properties of the nucleus: ${ }^{3}$ 1951.

Presented to the Society, April 28, 1951; received by the editors September 6,

1 This work was completed under the stimulating guidance of Professor R. $\mathrm{H}$. Bruck, at the University of Wisconsin, and is part of the author's doctoral dissertation.

2 Proceedings of the American Mathematical Society vol. 2 (1951) pp. 878-890.

${ }^{3}$ See BK Lemma 3.2 and R. D. Schafer, Representations of alternative algebras, Trans. Amer. Math. Soc. vol. 72 (1952) pp. 1-17, Lemmas 2, 3. 
LEMMA 2.3. Let $R$ be an alternative ring, $w, x, y, z$ arbitrary elements of $R$ and $n$ an arbitrary element of the nucleus $N$ of $R$. Then (i) $f(N, R, R, R)=0$, (ii) $(N, R) \subset N$, (iii) $(n x, y, z)=(x n, y, z)=n(x, y, z)$ $=(x, y, z) n$, (iv) $(x, y, z)(w, n)=-(w, y, z)(x, n),(\mathrm{v})((R, R, R), R, R)$ $(R, N)=0$.

\section{Simplicity.}

Lемма 3.1. Let $R$ be a non-associative, alternative, simple ring. Let $S$ be the set of all $s$ in $R$ with the property $s R=0=R s$. Then $S=0$. Equivalently, $R$ has no absolute divisors of zero.

Proof. Clearly $S$ is a two-sided ideal. If $S=R$ then the product of any two elements of $R$ is zero. But then $R$ is associative, a contradiction. Hence $S=0$ and the proof is complete.

THEOREM 3.2. Let $R$ be a non-associative, alternative, simple ring. Then its nucleus $N$ and centre $C$ coincide.

Proof. Obviously $C \subset N$. It remains to be shown that $N \subset C$. We define $T$ as the set of all $t$ in $R$ such that $t(N, R)=0=(N, R) t$. Let $r, r^{\prime}$ be in $R, n$ in $N, t$ in $T$. Then by Lemma 2.3 (ii), $t r \cdot\left(n, r^{\prime}\right)=t$ $\cdot r\left(n, r^{\prime}\right)=t\left(n, r r^{\prime}\right)-t(n, r) r^{\prime}+t \cdot 3\left(n, r, r^{\prime}\right)=0$. Similarly $r t\left(n, r^{\prime}\right)$ $=\left(n, r^{\prime}\right) t r=\left(n, r^{\prime}\right) r t=0$. Hence $T$ is a two-sided ideal. If $T=R$ then all $(N, R)$ are absolute divisors of zero, hence zero by Lemma 3.1, and the theorem is proven. The only remaining possibility is $T=0$. We now show this leads to a contradiction.

By Lemma 2.3 (v) we get that $((R, R, R), R, R)$ is in $T$, hence zero. This implies $(R, R, R) \subset N$. Using the exchange principle of Lemma 2.3 (iv) we note that the product of two associators is zero if at least two of the six components are equal. In particular let us choose $u=(x, y, z) \neq 0$. Then

$$
u^{2}=0 \text {. }
$$

Also $u r u=u r(x, y, z)=u(x r, y, z)-u(r, y, z) x-u f(x, r, y, z)$ $=-u f(x, r, y, z)$. But $-u f(x, r, y, z)=-u((x, r), y, z)-u(x, r,(y, z))$ $=0$. Thus

$$
u r u=0 \text { for all } r \text { in } R .
$$

Now define $A$ as the set of all $a$ in $R$ such that $a(u, R)=0=(u, R) a$. As above, it can be shown that $A$ is a two-sided ideal of $R$. Also $u$ is in $A$ by (3.1) and (3.2). Therefore $A=R$. Thus $(u, R)$ are absolute divisors of zero, hence zero. This implies $u$ is in $C$. The set of all $u r+i u$, where $r$ is in $R$ and $i$ is integral, is a two-sided ideal which must be all of $R$. But using (3.1) and (3.2) we obtain $R^{2}=0$, a con- 
tradiction. This completes the proof.

A theorem by $\mathrm{N}$. Jacobson ${ }^{4}$ states that $C$ is either 0 or a field. By Theorem 3.2 we now have the result that $N$ is either 0 or a field.

LEMMA 3.3. Let $R$ be a simple, non-associative, alternative ring. Then $(a, b, R)=0$ implies $(a, b)=0$.

Proof. Let $r, r^{\prime}$ be in $R$. Then $0=\left(r r^{\prime}, a, b\right)=f\left(r, r^{\prime}, a, b\right)$ $=\left(\left(r, r^{\prime}\right), a, b\right)+\left(r, r^{\prime},(a, b)\right)=\left(r, r^{\prime},(a, b)\right)$. Hence $(a, b)$ is in $N$. Also $(a, b, R)=0$ implies that $(a, a b, R)=0$. Therefore $(a, a b)=a(a, b)$ is in $N$. Suppose that $(a, b) \neq 0$. Then $C$ contains $(a, b)$, thus is a field. Consequently $a$ is in $C$, a contradiction. This proves the lemma.

\section{The main theorem.}

Lemma 4.1. Let $R$ be an alternative ring in which $(x, y)=0$ if and only if $(x, y, R)=0$. Let $K$ be an arbitrary subset of $R$ and define $T$ as the set of all $t$ in $R$ such that $t(K, R, R)=(K, R, R) t=(K, R t, R)$ $=(K, t R, R)=(K, t, R)=0$. Then $T$ is a two-sided ideal of $R$.

Proof. Choose $r, r^{\prime}, r^{\prime \prime}$ in $R, k$ in $K, t$ in $T$. Since $t\left(k, r^{\prime}, r^{\prime \prime}\right)=0$ $=\left(k, r^{\prime}, r^{\prime \prime}\right) t$, we have $\left(t,\left(k, r^{\prime}, r^{\prime \prime}\right)\right)=0$, so that

$$
(T,(K, R, R), R)=0 \text {. }
$$

From the hypothesis we obtain $(k, r t)=(k, t r)=0=(k, t)$. When we apply (BK2.6) we get $t(k, r)=0=(k, r) t$. Hence $(t,(k, r))=0$ and

$$
((K, R), T, R)=0 \text {. }
$$

Also $t r^{\prime} \cdot\left(k, r^{\prime \prime}\right)=t \cdot r^{\prime}\left(k, r^{\prime \prime}\right)=t\left(k, r^{\prime} r^{\prime \prime}\right)-t\left(k, r^{\prime}\right) r^{\prime \prime}-3 t\left(k, r^{\prime \prime}, r^{\prime}\right)=0$, using (BK2.6), (4.2), and hypothesis. Similarly $\left(k, r^{\prime \prime}\right) t r^{\prime}=0$ $=\left(k, r^{\prime \prime}\right) r^{\prime} t=r^{\prime} t\left(k, r^{\prime \prime}\right)$. This implies $R T(K, R)=T R(K, R)$ $=(K, R) R T=(K, R) T R=0$ and, as before,

$$
(T R,(K, R), R)=0=(R T,(K, R), R) .
$$

By hypothesis $\left(k, r t, r^{\prime} r^{\prime \prime}\right)=\left(k, t r, r^{\prime} r^{\prime \prime}\right)=0$. Applying $f$ to both associators yields

$$
f(K, T R, R, R)=0=f(K, R T, R, R) .
$$

Similarly from (4.2) we deduce

$$
f((K, R), T, R, R)=0 .
$$

Therefore $0=\left(t r,\left(k, r^{\prime}\right), r^{\prime \prime}\right)=f\left(t, r,\left(k, r^{\prime}\right), r^{\prime \prime}\right)+r\left(t,\left(k, r^{\prime}\right), r^{\prime \prime}\right)$ $+\left(r,\left(k, r^{\prime}\right), r^{\prime \prime}\right) t=\left(r,\left(k, r^{\prime}\right), r^{\prime \prime}\right) t$, using (4.3), (4.5), and (4.2).

${ }^{4}$ See N. Jacobson, Structure theory of simple rings without finiteness assumptions, Trans. Amer. Math. Soc. vol. 57 (1945) pp. 228-245. 
Similarly $t\left(r,\left(k, r^{\prime}\right), r^{\prime \prime}\right)=0$ and we write

$$
((K, R), R, R) T=0=T((K, R), R, R) .
$$

Since the hypothesis implies $T(K, R,(R, R))=0=(K, R,(R, R)) T$,

$$
T f(K, R, R, R)=0=f(K, R, R, R) T \text {. }
$$

Now $\left(k, r, r^{\prime}\right) \cdot r^{\prime \prime} t=\left(k, r, r^{\prime}\right) r^{\prime \prime} \cdot t=\left(k, r, r^{\prime \prime} r^{\prime}\right) t-f\left(k, r, r^{\prime \prime}, r^{\prime}\right) t$ $-r^{\prime}\left(k, r, r^{\prime \prime}\right) t=0$, using (4.1), (4.7), and hypothesis, while $\left(k, r, r^{\prime}\right)$ $\cdot t r^{\prime \prime}=\left(k, r, r^{\prime}\right) t \cdot r^{\prime \prime}=0$, using (4.1) and hypothesis. In this manner we obtain

$$
\begin{aligned}
(K, R, R) T R & =(K, R, R) R T=R T(K, R, R) \\
& =T R(K, R, R)=0 .
\end{aligned}
$$

Also $\left(r t \cdot r^{\prime}, r^{\prime \prime}, k\right)=f\left(r t, r^{\prime}, r^{\prime \prime}, k\right)+r^{\prime}\left(r t, r^{\prime \prime}, k\right)+\left(r^{\prime}, r^{\prime \prime}, k\right) r t=0$, because of (4.4), (4.8), and hypothesis. Similar arguments suffice to show $R T \subset T$ and $T R \subset T$. This completes the proof of the lemma.

THEOREM 4.2. Let $R$ be a non-associative, alternative, simple ring without nilpotent elements and of characteristic not 2. Then a necessary and sufficient condition that an element $c$ of $R$ be in the centre $C$ of $R$ is that there exist $a, b$ in $R$ such that $(a, b) \neq 0$ and $(c, a)=(c, b)=0$.

Proof. Combining Theorem 2.2 and Lemma 3.3 we know that $(x, y)=0$ if and only if $(x, y, R)=0$, for all $x, y$ in $R$. Since $R$ is not associative there exist $a, b$ in $R$ such that $(a, b) \neq 0$ and hence the condition is certainly necessary. Next we show that the condition is also sufficient. In Lemma 4.1 we let $K$ be the element $c$. From BK Lemma 3.3 it may be deduced that $(a, b)$ belongs to $T$. Since $(a, b)$ $\neq 0, T=R$ and hence $(c, R, R)=0$. Therefore, by Theorem $3.2, c$ is in the centre of $R$ and the proof is complete.

At this point we are ready to prove Theorem M. Let $R$ satisfy the hypothesis of Theorem M. Choose $u=(a, b, c) \neq 0$, where $a, b, c$ are in $R$. Then $u^{2} \neq 0$. By Lemma $2.1,\left(u^{2}, a, b\right)=0$ and by Theorem $2.2,(a, b) \neq 0$. Therefore Theorem 4.2 implies that $u^{2}$ is in the centre $C$ of $R$ and hence $C$ is a field. Let $x$ be any element in $R$ but not in $C$. Then by Theorem 3.2 there exist $y, z$ in $R$ such that $(x, y, z) \neq 0$. Using Theorem 2.2 we obtain $p x^{2}-q x+r=0$, where $p \neq 0$ and $r \neq 0$. Moreover, by Theorem 4.2, $p, q$, and $r$ are in the centre $C$ of $R$. Therefore $x^{-1}=-r^{-1} p x+r^{-1} q$ and $x x^{-1}=1=x^{-1} x$. Hence $\left(x, x^{-1}, R\right)$ $=0$. Consequently $x$ has a unique inverse and $R$ is a division ring, hence a Cayley-Dickson division algebra over its centre.

UNIVERSITY OF WISCONSIN 